
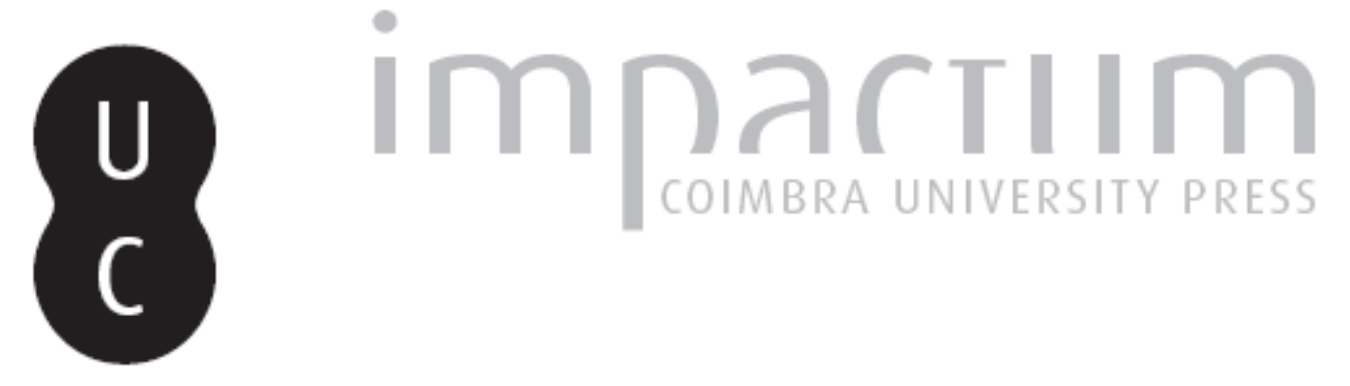

\title{
Compte rendu de II disordine ordinato, la filosofia dialettica di Platone
}

\author{
Autor(es): Lanoue, André
}

Publicado por: Imprensa da Universidade de Coimbra

URL persistente:

URI:http://hdl.handle.net/10316.2/47025

DOI:

DOI:https://doi.org/10.14195/2183-4105_18_11

Accessed : $\quad$ 26-Apr-2023 13:45:26

A navegação consulta e descarregamento dos títulos inseridos nas Bibliotecas Digitais UC Digitalis, UC Pombalina e UC Impactum, pressupõem a aceitação plena e sem reservas dos Termos e Condições de Uso destas Bibliotecas Digitais, disponíveis em https://digitalis.uc.pt/pt-pt/termos.

Conforme exposto nos referidos Termos e Condições de Uso, o descarregamento de títulos de acesso restrito requer uma licença válida de autorização devendo o utilizador aceder ao(s) documento(s) a partir de um endereço de IP da instituição detentora da supramencionada licença.

Ao utilizador é apenas permitido o descarregamento para uso pessoal, pelo que o emprego do(s) título(s) descarregado(s) para outro fim, designadamente comercial, carece de autorização do respetivo autor ou editor da obra.

Na medida em que todas as obras da UC Digitalis se encontram protegidas pelo Código do Direito de Autor e Direitos Conexos e demais legislação aplicável, toda a cópia, parcial ou total, deste documento, nos casos em que é legalmente admitida, deverá conter ou fazer-se acompanhar por este aviso.

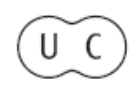


0

Established 1989

http://platosociety.org/

\section{Papers}

Christopher Buckels,

Aikaterini Lefka,

\section{Dossier}

Nicholas Baima,

Lloyd P. Gerson,

Sophia Stone,

Olivier Renaut.

Emily Katz,

Nicholas D. Smith,

Andy German,

-
$\varangle$
$Z$
0
-
$\vdash$
$\varangle$
$Z$
$\simeq$
$\amalg$
$\vdash$
$Z$
$\square$

Book Reviews

Anthony Preus,

André Lanoue,
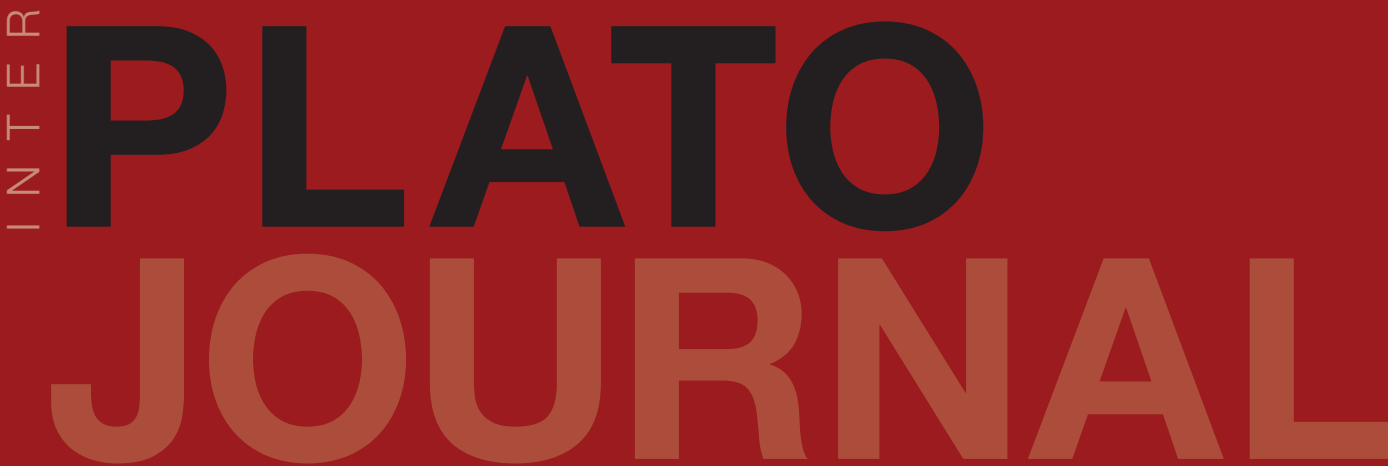

Société Platonicienne Internationale

Associazione Internazionale dei Platonisti

Sociedad Internacional de Platonistas

Internationale

Platon-Gesellschaft

Imprensa da

Universidade

de Coimbra

Coimbra

Universiy

Press 


\section{Compte rendu de II disordine ordinato, la filosofia dialettica di Platone, Maurizio Migliori, Morcelliana, 2013}

\author{
André Lanoue \\ Heidelberg Universtät \\ lanoue@stud.uni-heidelberg.de
}

Placée sous l'intitulé Il disordine ordinato: la filosofia dialettica di Platone, la monographie de Maurizio Migliori, parue chez Morcelliana en 2013, s'impose autant par ses dimensions monumentales (deux volumes qui recouvrent 1484 pages) que par sa visée "d'une autre époque» (p.8), qui consiste à proposer une vision unitaire de l'ensemble de la pensée platonicienne plutôt que d'en cibler un aspect spécifique. Ainsi, Migliori se penche sur l'écriture platonicienne, sa métaphysique, sur des questions de chronologie, de cosmologie, de psychologie, d'éthique et de politique en adhérant aux prémisses herméneutiques de l'École de Tübingen: la critique platonicienne de l'écriture expliquerait le fait que certains enseignements sont restés oraux; en conséquence, Platon miserait souvent sur l'aspect dramaturgique ou narratif des dialogues pour suppléer à la communication explicite qu'il ne se permettrait pas. Migliori se penche longuement sur la méthode littéraire de Platon dans le cadre du premier chapitre: Comment écrit Platon. Il introduit l'idée d'une «écriture contrôlée qui décide toujours quelle information fournir» (p. 41) et qui opère «une sorte de sélection du lecteur» (p. 39). S’il y a sélection du lecteur, Migliori n'en récuse pas moins le label «ésotériste», tout en se disant un exposant de l'École de Tübingen-Milan.

Devant un ouvre aux dimensions si gigantesgues, le meilleur parti, à notre sens, est de circonscrire certains points forts qui en véhiculent l'esprit. Deux aspects du premier chapitre nous semblent propices à une telle entreprise. La question de l'écriture contrôlée sera récurrente tout au long de l'œuvre et prendra, la plupart des fois, la forme du «jeu» dramaturgique par l'entremise duquel Platon inviterait ses lecteurs. La question de la doxographie aristotélicienne jette pour sa part les fondements de l'approche herméneutique de Migliori et appert, pour cette raison, de la première importance. 


\section{LA QUESTION DES TÉMOIGNAGES ARISTOTÉLICIENS}

Migliori se prononce en faveur d'une utilisation prudente des témoignages d'Aristote, pour laquelle il pose par conséquent quelques jalons de méthode. Il se positionne en ce sens par rapport à Brisson et Isnardi Parente, selon lesquels la doxographie aristotélicienne se référerait aux développements académiques de la doctrine platonicienne davantage qu'à la doctrine de Platon. Celle-ci serait dès lors, tout compte fait, une déformation de la théorie platonicienne et, par conséquent, sa valeur historique serait discutable. Leur vocabulaire est changé, le ton est moins tranchant, mais l'argument de Migliori, fort simple, consiste à mettre en lumière ce que présuppose leur position, qui, au bout du compte, reconduit grosso modo à celle de Cherniss; car il s'agit encore et toujours de plaider un doute raisonnable afin de mettre un embargo sur le témoignage d'Aristote. L'argumentation de Migliori souligne que, puisque les enseignements oraux sont souvent nommément attribués à Platon, pour que cette position soit valable, il faudrait tout d'abord admettre qu'Aristote eût menti, mais surtout, qu'il eût menti sans qu'on ne le perçât immédiatement à jour, ce qui appert très improbable: du Lycée à l'Académie, la chose se serait très certainement ébruitée assez vite. Migliori concède naturellement que Brisson a raison lorsqu'il mentionne que l'idée selon laquelle Platon réservait certains enseignements à l'oralité ne «ne se fonde sur aucune donnée incontestable ni chez Platon ni chez Aristote» (p. 42). L'histoire de la philosophie ne relève toutefois pas, de poursuivre Migliori, de certitudes incontestables, mais bien de «reconstructions plus ou moins fortes, plus ou moins complètes, plus ou moins cohérentes, plus ou moins probables» (idem). En ce sens, parce que les deux hypothèses relèvent du possible, $\mathrm{Mi}$ gliori préconise une méthode prudente: on peut

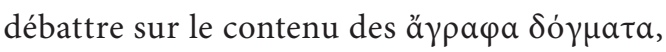
mais pas sur leur existence. En effet, si Aristote, dans le cadre de ses cours, pouvait librement parler de doctrines non écrites, c'est que l'on devait bien savoir à quoi il faisait allusion. En vue d'une reconstruction des enseignements oraux, il sied toutefois d'analyser systématiquement le langage d'Aristote: lorsqu'il s'exprime en adoptant la terminologie platonicienne, il serait injustifié de le remettre en cause; mais s'il emploie plutôt son propre vocabulaire, la doctrine platonicienne a nécessairement subi une transposition qui dépasse la simple sphère langagière et touche également au contenu théorique. Par rapport à l'aspect polémique, il est admis qu'Aristote, en tant que théoricien, tente d'attaquer Platon sur le point qu'il considère le plus vulnérable, quitte à légèrement remanier son propos afin de faire pencher la balance en sa faveur. Il faut néanmoins garder à l'esprit, précise Migliori, que si Aristote avait trop modifié les thèses de Platon, il aurait en premier lieu couru le risque qu'on le lui reprochât ouvertement, ce que Speusippe, Xénocrate ou d'autres n'auraient sans doute pas manqué de faire. Deuxièmement, il est très probable que, si Arisote s'était permis de remanier la position platonicienne en profondeur, c'est à sa propre argumentation qu'il aurait nui en la discréditant.

L'argumentation de Migliori, sur ce point, nous semble irréprochable.

\section{LE «JEU DE L'ÉCRIT»}

C'est parce que Platon fait jour de ses réticences vis-à-vis de l'écrit qu'intervient la notion du «jeu». Ce jeu prendrait plusieurs 
formes et viserait toujours à s'adapter à l'âme du lecteur. Il s'agirait de:

fournir une aide respecteuse du niveau de maturité de l'élève, dire de manière simple pour les âmes simples et complexe pour les âmes complexes, ce qui requiert toujours et donc justifie une grande réticence éducative du maître, qui ne veut empêcher le lecteur de découvrir la vérité en la lui communiquant (italiques de l'auteur, p. 72).

Platon, parce qu'il aurait voulu écrire en respectant ses principes pédagogiques, aurait:

inventé une technique d'écriture qui lui permettrait d'affronter les deux principaux problèmes: la croissance personnelle $\mathrm{du}$ sujet et la défense du contenu qu'il met à disposition du lecteur (italiques de l'auteur, p.74).

Migliori explique donc l'approche narratologique par sa vocation parénétique, de sorte que la diversité des procédés littéraires et de la mise en scène dans les dialogues est ultimement reliée à la diversité des âmes des lecteurs. Il sera du reste soutenu que, "vu le génie artistique de Platon, il serait plus judicieux de ne pas essayer de reconduire en un schéma unique sa capacité à communiquer » (italiques de l'auteur, p.93). On remarque là une approche nuancée qui consiste à ne pas fixer dans une structure trop rigide la versatilité de la dramaturgie platonicienne. En ce sens, plutôt que d'attribuer au seul dialecticien le monopole de la position platonicienne, ce qui reviendrait rechercher dans les dialogues la forme du traité, Migliori prône une herméneutique plus ouverte

La fonction des interlocuteurs ne sont pas seulement les porteurs de leur propre position, mais peuvent aussi devenir les porte-paroles d'une position correcte ou d'un signal que l'Auteur entend donner (italiques de l'auteur, p. 94).

Migliori prône donc qu'on laisse parler la dramaturgie afin de jouer le jeu auquel Platon invite ses lecteurs. La visée de Platon serait toujours (partiellement du moins) de nature protreptique, en tant qu'elle aspirerait constamment à opérer le retournement de l'âme qu'est la philosophie (un dialogue comme le Parménide ne serait évidemment pas proptreptique sous le même rapport qu'un dialogue comme l'Apologie de Socrate). Ces jeux peuvent d'ailleurs s'étaler sur plusieurs dialogues, pensons entre autres à la trilogie Sophiste-Politique-Philosophe où le point culminant de la trilogie, par choix de l'Auteur, serait demeuré non écrit. L'intention de Platon aurait été de se limiter à «conduire son lecteur le plus près possible de ce niveau de réflexion <celui de s'interroger, à la lumière des acquis du Sophiste et du Politique, sur l'essence du philosophe $>»$ (p.113), sans pour autant lui exposer sa doctrine explicitement, ce qui n'aurait pas représenté une approche pédagogiquement viable.

Migliori, qui multiplie les exemples de jeux dramaturgiques, se limite néanmoins à illuster au moyen d'exemples et ne fixe pas la dramaturgie platonicienne dans un schéma structurant, qui serait nécessairement trop peu malléable pour rendre justice à la richesse littéraire des dialogues. Au contraire, il lui insuffle quelques principes de cohésion: la critique platonicienne de l'écriture, le témoignage de l'Ancienne Académie et la visée protreptique de Platon, sur la base desquels il élabore la notion d'un «jeu» structurant la narrativité des dialogues. Cette approche peut être critiquée en cela qu'elle serait une license pour l'interprétation ad hoc, et par suite un retour au point de départ. Nous répondrions alors que si l'on 
admettait ces quelques principes pour l'interprétation des dialogues, tout en soutenant que les dialogues sont et demeurent la voie la plus sûre pour comprendre la philosophie de Platon, l'effort herméneutique contemporain serait déjà amplement plus unitaire. Migliori parle d'une «écriture contrôlée» et donne une quantité impressionnante d'exemples qui démontrent qu'il y a bel et bien un constant contrôle de l'information fournie de la part de Platon. Si l'approche herméneutique qu'il privilégie est assez libre, nous aimerions la désigner comme une «herméneutique contrôlée», qui encadre la lecture sans pour autant brimer sa marge de manœuvre ni réduire l'immensité fluide du corpus platonicien à un schéma qui, pour brillant qu'il soit, serait toujours trop restrictif. Par son étude rigoureuse de la dramaturgie platonicienne, le dernier ouvrage de Migliori offre qui plus est une énième réfutation à ceux qui estiment qu'«une lecture ésotériste vide les dialogues, et surtout les dialogues de jeunesse de leur contenu spécifique, pour les représenter sous le jour d'une doctrine des principes» (Brisson 1998, p. 494). Pour la vaste étendue des sujets traités, l'originalité de sa lecture et surtout, pour ses thèses minutieusent étayées, Il disordine ordinato, la filosofia dialettica di Platone constitue, à notre avis, l'une des études spécialisées les plus importantes du vingt-etunième siècle et promet de s'imposer en incontournable des études platoniciennes. 